\section{Exercise and Antioxidant In- take in Aging Normotensive and Hypertensive Individuals}

\author{
Mercier $\mathrm{S}^{1,2}$, Khurana $\mathrm{S}^{4}$, Larivière $\mathrm{C}^{1,4,5} \mathrm{Tai}^{\mathrm{T}} \mathrm{TC}^{2,4,5 *}$ and \\ Venkataraman $\mathbf{K}^{3 *}$
}

${ }^{1}$ School of Human Kinetics, Laurentian University, Canada
${ }^{2}$ Chemistry and Biochemistry, Laurentian University, Canada
${ }^{3}$ Gerontology Department, Huntington University, Canada

${ }^{4}$ Medical Sciences Division, Northern Ontario School of Medicine, Canada

${ }^{5}$ Biomolecular Sciences Program, Laurentian University, Canada

\begin{abstract}
Oxidative stress has been implicated in both aging and the development of pathological cardiovascular illness. Exercise's role in the management of hypertension is well established and antioxidant supplementation may be beneficial to individuals with chronic health conditions. In combination, the effects of antioxidants and physical activity are controversial. Some studies have suggested that antioxidant supplementation inhibits exercise's protective modulation of endogenous antioxidant enzymes.

There is less research on the combined effects of exercise and antioxidant supplementation in older hypertensive individuals, who are under increased oxidative stress. The aim of our study was to further examine associations between antioxidant intake and exercise in the aetiology of hypertension in older adults. Hypertensive status, weekly physical activity levels and daily antioxidant consumption levels were assessed using questionnaires. We hypothesized that a greater number of normotensive individuals would report both adequate physical activity and adequate antioxidant consumption than hypertensive individuals. While endogenous antioxidants were not examined, results from this study were able to establish trends in antioxidant consumption and physical activity levels and a significant correlation between vitamin A consumption and hypertensive status. Keywords: Cardiovascular disease; Diet; Physical activity
\end{abstract}

\section{Introduction}

Cardiovascular Disease (CVD) is a leading cause of death worldwide $[1,2]$. It is characterized by a diseased state of the heart

*Corresponding authors: Krishnan Venkataraman, Gerontology Department, Huntington University, 935 Ramsey Lake Road, Sudbury P3E 2C6, Canada, Tel: +1 7056734126 Extn: 218; Fax: +1 705673 6917; E-mail: kvenkataraman@huntingtonu.ca

T.C. Tai, Medical Sciences Division, Northern Ontario School of Medicine, Laurentian University, 935 Ramsey Lake Road, Sudbury P3E 2C6, Canada, Tel: +1 7056627239; Fax: +1 7056754858; E-mail: tc.tai@nosm.ca

Citation: Mercier S, Khurana S, Larivière C, Tai TC, Venkataraman K (2016) Exercise and Antioxidant Intake in Aging Normotensive and Hypertensive Individuals. J Gerontol Geriatr Med 2: 010

Received: July 27, 2016; Accepted: November 23, 2016; Published: December 07, 2016 and/or blood vessels and encompasses a wide range of chronic and acute conditions, including atherosclerosis, ischemia, angina, myocardial infarction, cerebrovascular disease and hypertension [3].

Hypertension itself is extremely widespread with one billion people affected worldwide, leading to an estimated million deaths annually [4]. Aging individuals are at a higher risk of developing hypertension than younger populations. In 2013, Statistics Canada reported a national average of over $45 \%$ of the population aged $65-74$ years (males and females) presenting with hypertension, compared to $30-$ $35 \%$ in the $55-64$ year and a dramatic increase from $15-25 \%$ of the population in the 45-54 year age categories. Below the age of 45 , the proportion of the population presenting with hypertension drops to under 10\% (Statistics Canada, 2013).

\section{The "Combined Antioxidant and Exercise Intervention" controversy}

Oxidative stress has been extensively implicated in the development of chronic illnesses. Both exercise and antioxidant supplementation have been suggested to have beneficial effects on cardiovascular health and reduce the risk of hypertension and cardiovascular disease. Exercise is a source of oxidative stress however, it has been established that the free radicals resultant of physical activity induce an up-regulation of the body's endogenous enzymatic antioxidant systems, thereby modulating the body's antioxidant defenses [5].

The health impact of antioxidant supplementation in combination with exercise in healthy individuals is controversial. In 2014, Draeger et al., performed a review of research on the effects of antioxidant supplementation on exercise-induced oxidative stress, concluding that benefits of taking antioxidant supplements to boost athletic performance remains to be verified, with studies demonstrating modest increases in performance with supplementation, no effects, or even detrimental effects [6].

\section{Studies in young adults}

There have been many studies on the effects of antioxidants on exercise induced oxidative stress, with widely varied conclusions. A 1997 study concluded that oxidative stress induced by exercise is improved with vitamin C supplementation [7]. Furthermore, supplementing the diets of athletes with vitamins $\mathrm{C}, \mathrm{E}$ and $\mathrm{B}$-carotene increased body levels of these antioxidants, though the levels were on par with those in people with nutrient-rich diets. Dietary supplementation improved lymphocytic antioxidant capacity as well as protection of erythrocyte glutathione reductase, which is known to decrease in response to a session of exercise [8].

A 2006 study demonstrated improvements in the degree of protein oxidation in trained cyclists (mean age $=23.7$ years) upon consumption of a drink with polyphenolic antioxidants, although neither the extent of lipid peroxidation nor the total plasmatic antioxidant capacity changed [9]. Although, flavonoids and cinnamic acid, the primary sources of polyphenol in the drink administered, are stronger antioxidants than alpha-tocopherol (vitamin E) or ascorbic acid (vitamin C), they appear to be poorly bioavailable in humans [10]; the authors 
provide this as an explanation for the lack of improvement in the body's "Total Antioxidant Capacity" (TAC), though they conclude that their results are indicative of a positive effect on exercise-induced oxidative damage [9].

Ristow et al., (2009) examined the effects of antioxidant supplementation on insulin sensitivity and endogenous antioxidant activity in untrained and pre-trained males aged 25-35. It was found that antioxidant intake actually inhibited the exercise produced ROS induced physiological improvements in insulin sensitivity and enzymatic antioxidant defense capacity [11].

\section{Studies in older adults}

Several studies demonstrate a correlation between persistent oxidative stress and development of chronic disease conditions, and its mitigation by exercise. Although exercise itself is a source of oxidative stress, there is considerable evidence for the beneficial effects of exercise. This paradox is somewhat complicated by mixed results obtained by combining exercise and antioxidant therapies, as highlighted below [5].

A 2002 study on elderly subjects found that neither exercise nor antioxidant supplementation alone had any effect on exercise induced oxidative stress, though physically active individuals exhibited a lower oxidative response to exercise in comparison to less active individuals [12]. In mildly hypertensive elderly (mean age: 71 years) patients, antioxidant supplements taken post-exercise negated the blood pressure improvements induced by exercise. Positive correlations between vitamin C [13] or resveratrol [14] and physical performance have been established, while vitamin E actually correlated negatively [15]. Another study found that vitamin $\mathrm{C}$ and $\mathrm{E}$ supplementation in conjunction with resistance training improved the antioxidant status of the plasma without ameliorating the pro-oxidant status [16]. A recent review (2011) highlights some important differences in response between younger and aging populations; for example, antioxidant supplementation improved blood perfusion and muscle phosphocreatine recovery in older, but not younger, individuals [5]. An analysis of the results of 1514 individuals over the age of 40 taken from the Hortega nutrition study showed no correlation between the dietary consumption of antioxidant molecules such as vitamin A, D and E on hypertensive status [17]. In young sedentary, older sedentary and older trained men, infused ascorbic acid improved the flow-mediated dilation of the brachial artery in sedentary older adults but not in the other two groups, suggesting that vitamin $\mathrm{C}$ may help to reverse arterial flow impairment and endothelial dysfunction by oxidative stress and preserve flow rate in active older adults [18]. Further, while infusion improved endothelial function, regular oral doses did not, suggesting that dietary supplementation alone may not reach critical ROS scavenging capacity. This is supported by another study examining oral dose of vitamin $\mathrm{C}$, concluding that endothelial dysfunction was not reversed by either acute or chronic treatment in hypertensive individuals [19].

\section{Study rationale and objectives}

Given the apparent role of oxidative stress in both aging and the development of pathological cardiovascular illness, it is possible that antioxidant supplementation may have a particularly beneficial effect in older adults with a chronic health condition such as hypertension [20]. There is considerably less research on the combined effects of exercise and antioxidant supplementation in elderly hypertensive individuals than in young, healthy individuals reflecting the need for further studies in this area. Our study aimed to further examine associations between antioxidant intake and exercise in the aetiology of hypertension in older adults. We hypothesized that a greater number of normotensive individuals would report both adequate physical activity and adequate antioxidant consumption compared to hypertensive individuals.

\section{Methods}

\section{Recruitment}

Subjects were recruited via social media and from Parkside recreation centre for older adults in Sudbury, Ontario. Information and study packages were provided to interested individuals fitting the age criterion via email and onsite. All individuals aged 50 years and older were invited to fill out questionnaires all individuals younger than 50 years were excluded from the study. The study was carried out between January to March, 2016.

\section{Ethics statement}

Approval of the project was received from the School of Human Kinetics Research Ethics Committee (a subset of Laurentian University's Research Ethics Board). Participants were asked to sign and return a copy of an informed consent form to the researcher, after which they were provided with further instruction and the study forms.

\section{Study protocols}

Participants were tasked with filling out two questionnaires and a four-day food journaling activity, during the month of February. The first questionnaire comprised of background questions pertaining to hypertensive status, family history, medications, vitamin supplementation, socioeconomic status and ease of accessibility to housing and leisure services. The second, the "Godin Leisure Time Activity Questionnaire (GLATQ)" [21], consisted of two questions. The GLATQ determined weekly physical activity levels, in Metabolic Equivalents (METs). The journaling activity required participants to keep a detailed record of their food and beverage consumption.

\section{Measurement and data analysis}

Questionnaires were used to derive information on hypertensive status (yes or no) and vitamin supplementation. GLATQ scores were computed using their scoring formula and dietary values for vitamins $\mathrm{A}, \mathrm{C}, \mathrm{E}$ and selenium were obtained by logging and averaging the recorded four days of food intake for each participant using Cronometer, an online nutrient tracker. Mean values for weekly physical activity and average daily intakes for vitamin A, vitamin C, vitamin E and selenium were graphed to highlight differences between groups using a statistical analysis program (Prism, GraphPad Software, Inc.).

A total of 22 subjects were enrolled in the study. The group divided into two cohorts hypertensive (7) and normotensive (15) and classified as either "Yes" or "No" in the following categories: 1) adequate physical activity, 2) adequate Vitamin A, 3) adequate Vitamin C, 4) adequate Vitamin E, and 5) adequate selenium. Vitamin supplements and smoking were taken into account. Respondents were grouped into contingency tables (Tables 1-3); these were analyzed for statistical significance using Barnard's test [22]. Both cohorts of participants were then subdivided into the following four categories and grouped into further contingency tables:

- Adequate Weekly PA AND Adequate Antioxidant Intake

- Adequate Weekly PA ONLY

- Adequate Antioxidant Intake ONLY

- Inadequate Weekly PA AND Inadequate Antioxidant Intake 


\begin{tabular}{|c|c|c|c|}
\hline Total (\% column) (\%line) & \multicolumn{2}{|c|}{ Adequate vitamin A consumed? } & \\
\hline Hypertensive? & Yes & No & \\
\hline \multirow{2}{*}{ Yes } & $1(9.1 \%)$ & $6(54.5 \%)$ & $7(31.8 \%)$ \\
\cline { 2 - 4 } & $(14.3 \%)$ & $(85.7 \%)$ & \\
\hline \multirow{2}{*}{ No } & $10(90.9 \%)$ & $5(45.5 \%)$ & $15(68.2 \%)$ \\
\cline { 2 - 4 } & $(66.7 \%)$ & $(33.3 \%)$ & \\
\hline & $11(50.0 \%)$ & $11(50.0 \%)$ & 22 \\
\hline
\end{tabular}

Table 1: Vitamin A consumption and hypertensive status.

\begin{tabular}{|c|c|c|c|}
\hline \multirow{2}{*}{$\begin{array}{c}\text { Total (\% column) (\%line) } \\
\text { Hypertensive? }\end{array}$} & \multicolumn{2}{|c|}{ Adequate vitamin $\mathrm{C}$ consumed? } & \\
\hline & Yes & No & \\
\hline \multirow[t]{2}{*}{ Yes } & $3(23.1 \%)$ & $4(44.4 \%)$ & $7(31.8 \%)$ \\
\hline & $(42.9 \%)$ & $(57.1 \%)$ & \\
\hline \multirow[t]{3}{*}{ No } & $10(76.9 \%)$ & $5(55.6 \%)$ & $15(68.2 \%)$ \\
\hline & $(66.7 \%)$ & $(33.3 \%)$ & \\
\hline & $13(59.1 \%)$ & $9(40.9 \%)$ & 22 \\
\hline
\end{tabular}

Table 2: Vitamin $\mathrm{C}$ consumption and hypertensive status.

\begin{tabular}{|c|c|c|c|}
\hline \multirow{2}{*}{$\begin{array}{c}\text { Total ( } \% \text { column) (\%line) } \\
\text { Hypertensive? }\end{array}$} & \multicolumn{2}{|c|}{ Adequate vitamin E consumed? } & \\
\hline & Yes & No & \\
\hline \multirow[t]{2}{*}{ Yes } & $1(20.0 \%)$ & $6(35.3 \%)$ & $7(31.8 \%)$ \\
\hline & $(14.3 \%)$ & $(85.7 \%)$ & \\
\hline \multirow[t]{3}{*}{ No } & $4(80.0 \%)$ & $11(64.7 \%)$ & $15(68.2 \%)$ \\
\hline & $(26.7 \%)$ & $(73.3 \%)$ & \\
\hline & $5(22.7 \%)$ & $17(77.3 \%)$ & 22 \\
\hline
\end{tabular}

Table 3: Vitamin E consumption and hypertensive status.

Adequate Weekly PA was defined as any value greater than 600 moderate/high intensity METs/min. These values were selected based on recommendations for adults by the World Health Organization's "Global Physical Activity Questionnaire" [23]. Inadequate Weekly PA was defined as anything lower than $600 \mathrm{METs} / \mathrm{min}$.

Adequate antioxidant intake was identified as scoring above adequate levels for any two of the four antioxidant factors (vitamin A, vitamin $\mathrm{C}$, vitamin $\mathrm{E}$ and selenium) evaluated:

- Adequate Vitamin A: $>10000$ IUs $^{*}$

- Adequate Vitamin C: $>75 \mathrm{mg}$ (female); > 90mg (male); Add $35 \mathrm{mg}$ for smokers

- Adequate Vitamin E: $\quad>15 \mathrm{mg}$

- Adequate Selenium: $>55 \mu \mathrm{g}$

These values were chosen based on dietary reference intake tables for vitamins and minerals provided by Health Canada [24].

\section{Results}

The study included twenty-two participants of which, seven self-reported as being hypertensive, while fifteen self-reported as being normotensive; socio-demographic data is shown in table 4 .

No significance was demonstrated for physical activity, vitamin C, vitamin $\mathrm{E}$ and selenium according to hypertensive status (Figures 1B1E). In contrast, Vitamin A consumption was found to be lower in the hypertensive group compared to the normotensive cohort (Wald Statistic $=2.289$, Nuisance parameter $=0.6601, p=0.013)($ Figure $1 \mathrm{~A})$.

\begin{tabular}{|c|c|c|}
\hline \multirow{2}{*}{ Variables } & \multicolumn{2}{|c|}{ Subjects } \\
\hline & $\mathrm{N}$ & $\%$ \\
\hline \multicolumn{3}{|l|}{ Sex } \\
\hline Male & 5 & 22.7 \\
\hline Female & 17 & 77.3 \\
\hline \multicolumn{3}{|l|}{ Age (years) } \\
\hline $50-60$ & 14 & 63.6 \\
\hline $61-70$ & 6 & 27.3 \\
\hline$>70$ & 2 & 9.09 \\
\hline \multicolumn{3}{|l|}{ Education } \\
\hline Incomplete high school & 2 & 9.09 \\
\hline High school & 2 & 9.09 \\
\hline Bachelors/Diploma & 14 & 63.6 \\
\hline Graduate or higher & 4 & 18.18 \\
\hline \multicolumn{3}{|l|}{ Smoking status } \\
\hline Y & 1 & 4.5 \\
\hline $\mathrm{N}$ & 21 & 95.45 \\
\hline \multicolumn{3}{|l|}{ Annual Income } \\
\hline$<44,000$ & 5 & 22.7 \\
\hline $44-88,000$ & 8 & 36.4 \\
\hline $88-136,000$ & 2 & 9.09 \\
\hline$>136,000$ & 2 & 9.09 \\
\hline Preferred not to answer & 5 & 22.7 \\
\hline
\end{tabular}

Table 4: Socio-demographic data of study participants regarding their sex age, education, income, and smoking status.

In the hypertensive cohort, trends towards lower physical activity levels and lower consumption of vitamins $\mathrm{C}$ and $\mathrm{E}$ were shown compared to the normotensive cohort (Figures 1C-1D). Selenium consumption was very modestly higher in the hypertensive group compared to the normotensive cohort (Figure 1E).

In the normotensive cohort, 6 of 15 participants (40.0\%) reported as achieving both adequate physical activity and antioxidant consumption (Table 5), 5 of 15 participants (33.3\%) reported adequate levels of antioxidant consumption only. 2 of 15 (13.3\%) reported as achieving adequate levels of physical activity only, and 2 of 15 (13.3\%) reported as achieving inadequate levels of both physical activity and antioxidant consumption. In total, $73.3 \%$ of normotensive respondents reported adequate antioxidant intake, while $53.3 \%$ reported adequate physical activity (Table 5 ). The above results are summarized in table 5.

In the hypertensive cohort, 0 of 7 participants $(0.0 \%)$ reported as achieving both adequate physical activity levels and adequate antioxidant consumption (Table 5). 1 in 7 (14.3\%) reported adequate antioxidant consumption only, while 4 in 7 (57.1\%) reported achieving adequate levels of physical activity only. 2 in 7 (28.6\%) achieved both inadequate levels of both physical activity and antioxidant consumption. In total, $14.0 \%$ of hypertensive respondents reported adequate antioxidant intake, while $57.1 \%$ of respondents reported adequate physical activity (Table 5).

\section{Discussion}

In our cohort, extremely high levels of vitamin A (identified as > 10,000 IU; Health Canada's present maximum recommended daily allowance) were significantly correlated to normotension (Figure 1), 


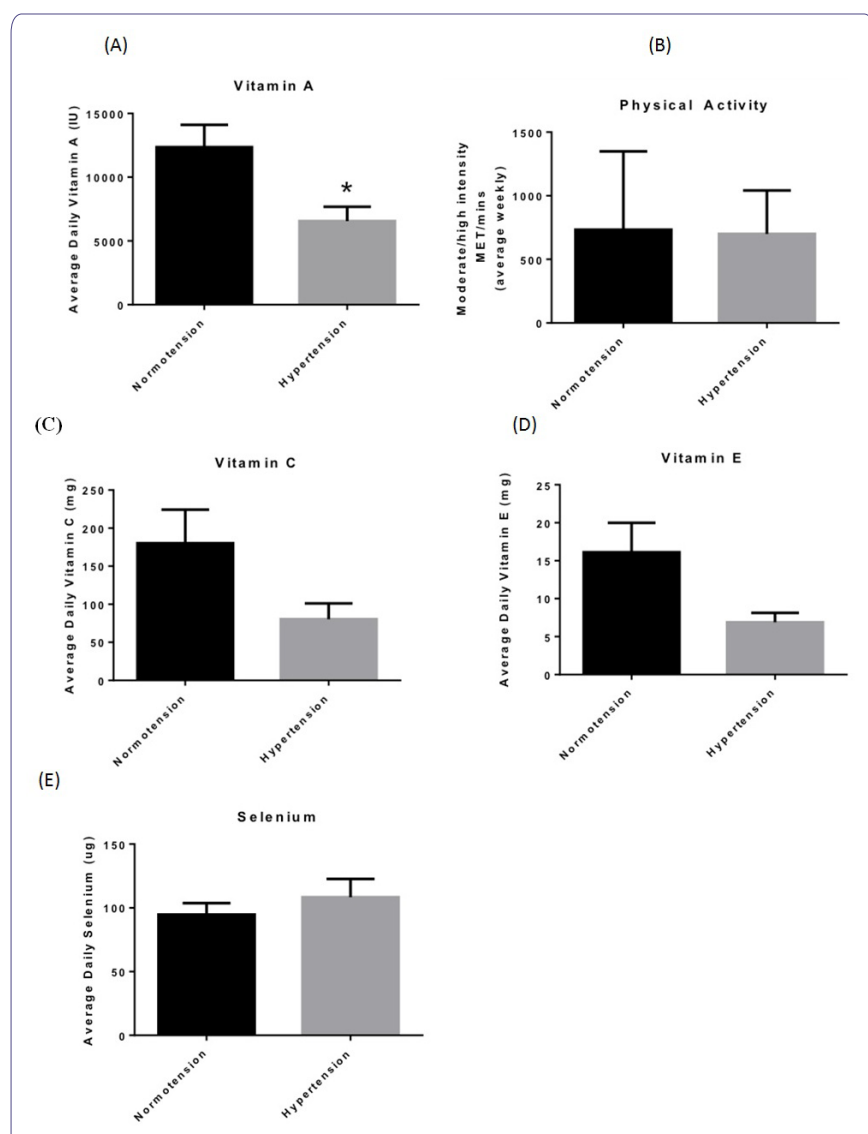

Figure 1: Antioxidant consumption, and physical activity measurements in normotensive and hypertensive subjects.

Data were calculated as presented in the methods, and represented as means \pm SEM for Vitamins A consumption (A), physical activity (B), Vitamin C intake (C), Vitamin E intake (D) and Selenium intake (E)

possibly reflective of long-term consumption of vitamin $\mathrm{A}$ as a factor implicated in the prevention of hypertension. Outside of this study, there is little longitudinal data suggesting a relationship between vitamin A consumption and hypertensive status. This finding may have been coincidental and due to the small sample size; further studies with a larger sample size would make this correlation more meaningful.

No significant results were established between hypertensive status and the consumption of vitamin C, vitamin E, selenium, or adequate levels of physical activity. However, with the exception of selenium,

\begin{tabular}{|c|c|c|}
\hline Groupings & Normotensive & \multirow{2}{*}{ Hypertensive (n= 7) } \\
\cline { 2 - 3 } & $\mathbf{( n = 1 5 )}$ & $0.0 \%$ \\
\hline $\begin{array}{c}\text { Adequate PA and Adequate } \\
\text { Antioxidant Intake }\end{array}$ & $40.0 \%$ & $14.0 \%$ \\
\cline { 2 - 3 } \begin{tabular}{c} 
Adequate Antioxidant Intake Only \\
Adequate Physical Activity Only \\
Inadequate PA and Antioxidant \\
\cline { 2 - 3 } Intake
\end{tabular} & $33.3 \%$ & $57.1 \%$ \\
\cline { 2 - 3 } & $13.3 \%$ & $28.6 \%$ \\
\hline Total Adequate Antioxidant Intake & $73.3 \%$ & $14.0 \%$ \\
\cline { 2 - 3 } Total Adequate Physical Activity & $53.3 \%$ & $57.1 \%$ \\
\hline
\end{tabular}

Table 5: Summary of Physical Activity and Antioxidant Consumption in the Normotensive and Hypertensive Cohorts (in percentages). a slight trend towards higher physical activity levels (Figure 1E) and trends towards greater consumption of vitamins $\mathrm{C}$ and $\mathrm{E}$ (Figures 1C and 1D) were shown in the normotensive cohort compared to the hypertensive cohort, findings which are relatively well supported by previous literature.

The contingency tables comparing physical activity levels and antioxidant levels in the normotensive (Table 6) and hypertensive (Table 7) cohorts provided interesting insight towards confirming our hypothesis. $40.0 \%$ of normotensive respondents reported achieving both adequate physical activity levels and adequate antioxidant consumption (6/15, Table 6). None of the hypertensive respondents achieved this result. We have established that, in our population, a greater percentage of normotensive individuals meet both antioxidant and physical activity recommendations compared to hypertensive individuals. These results are summarized in table 5 .

When examining physical activity alone, $53.3 \%$ of normotensive and $57.1 \%$ of hypertensive individuals reported achieving an adequate weekly amount of medium-to-high intensity physical activity in a week. While we saw an overall higher physical activity level of the normotensive group, the proportion of individuals achieving adequate levels of physical activity as defined by the WHO [23] was not

\begin{tabular}{|c|c|c|c|}
\hline \multirow{2}{*}{ Total (\% column) (\%line) } & \multicolumn{2}{|c|}{ Adequate physical activity? } & \\
\hline Adequate antioxidant intake? & Yes & No & \\
\hline Yes & $6(75.0 \%)$ & $5(71.4 \%)$ & $11(73.3 \%)$ \\
\cline { 2 - 4 } & $(54.5 \%)$ & $(45.5 \%)$ & \\
\hline No & $2(90.9 \%)$ & $2(45.5 \%)$ & $4(26.7 \%)$ \\
\cline { 2 - 4 } & $(50.0 \%)$ & $(50.0 \%)$ & \\
\hline & $8(53.3 \%)$ & $7(46.7 \%)$ & 15 \\
\hline
\end{tabular}

Table 6: Physical activity and antioxidant intake in the normotensive cohort.

\begin{tabular}{|c|c|c|c|}
\hline Total (\% column) (\%line) & \multicolumn{2}{|c|}{ Adequate physical activity? } & \\
\hline Adequate antioxidant intake? & Yes & No & \\
\hline Yes & $0(0.0 \%)$ & $1(33.3 \%)$ & $1(14.3 \%)$ \\
\cline { 2 - 4 } & $(0.0 \%)$ & $(100.0 \%)$ & \\
\hline No & $4(100 \%)$ & $2(66.7 \%)$ & $6(85.7 \%)$ \\
\hline & $(100.0 \%)$ & $(33.3 \%)$ & \\
\hline & $4(57.1 \%)$ & $3(42.9 \%)$ & 7 \\
\hline
\end{tabular}

Table 7: Physical activity and antioxidant intake in the hypertensive cohort.

different between our hypertensive and normotensive cohorts. While not a cure for hypertension, exercise has been shown to help manage blood pressure. It is encouraging to see that a similar number of hypertensive and normotensive individuals are engaging in physical activity, although it is less positive to note that nearly half of our respondents reported activity levels below recommended amounts. When examining antioxidant intake, $73.3 \%$ of normotensive achieved adequate daily antioxidant consumption (11/15, Table 6), compared to only $14.3 \%(1 / 7$, Table 7$)$ of individuals in the hypertensive group. Although this result may be coincidental, it supports the idea that consumption of dietary antioxidants may be beneficial in maintaining normal blood pressure.

Our study cohort was relatively small as such; it was difficult to establish more than tentative relationships between hypertension and any of the study variables. Given the small sample size, we were unable to establish any significant relationships specific to both physical 
activity and antioxidant consumption in the context of hypertension however, certain trends were apparent.

\section{Limitations}

The major limitation of this study was its sample size. At present, this investigation best serves as a strong pilot study. Values obtained in the exercise and antioxidant categories varied widely from individual to individual at times, leading to large standard deviations. In general, a larger sample size would lead to more powerful results and the possibility of drawing further conclusions.

The accuracy of results may have been confounded by the self-reporting nature of the study people notoriously under report their food consumption and misclassify the type and intensity of the exercise they perform [25-28]. The overall scope of diet assessed in this study (four days in the month of February) was extremely limited. The types of foods humans consume tend to vary seasonally [29] and individuals in winter climates have less access to (and thereby consume less) fresh produce. This of itself has an impact on overall antioxidant consumption. Other options for dietary assessment, such as a Food Frequency Questionnaire (FFQ), were also explored in the study design however, preliminary tests using FFQs designed to analyse an entire year's food consumption proved to take an "unreasonably long time" (i.e., in excess of two hours). In order to make the study more accessible, the food diary was implemented in lieu.

It was important to consider that some participants may be hypertensive and be unaware of that fact. All hypertensive individuals participating in the study reported as being on antihypertensive medications. Many cases of hypertension also have a large genetic component [30-32] hypertensive status not correlative to diet or activity levels may be attributable to hereditary factors.

Outside of the antioxidants assessed in this study, there are other forms of dietary antioxidants with potentially interesting implications pertaining to chronic health. This study did not assess dietary molecules such as polyphenols, which have recently been under investigation as possessing potential health-benefits $[33,34]$.

This study also highlighted the need for food questionnaires targeted to antioxidant intake. As of this writing, the only FFQs targeted to antioxidant consumption $[35,36]$ are still in the process of being validated. Additional considerations when working with very young or older populations include the length of the questionnaire and complexity of the questions being asked. A FFQ specifically targeted towards and accessible to older populations would be particularly beneficial to a study of this nature, as appetite and nutritional status tend to decline with age [37].

\section{Implications}

This study has identified a potential area of interest that of vitamin A consumption in the context of hypertension. While still requiring further exploration, this study might lay the groundwork for future animal and epidemiological studies. The current preliminary data associate adequate physical activity and adequate antioxidant intake together with normal blood pressure. In our study cohort, higher antioxidant intake was of particular note in the normotensive cohort relative to the hypertensive cohort.

While no clear links between hypertensive status and activity levels were established in this study, the benefits of physical activity in aging populations are well documented [38-41]; regardless, it is known that physical activity levels typically decrease with age for various reasons [42]. In our study cohort, it is interesting to note that half of the participants (both hypertensive and normotensive) were below the World Health Organization's recommended daily physical activity levels, despite all participants reporting having adequate access to leisure and recreation services in the background information questionnaire.

\section{Future Research}

As mentioned above, little research exists with regards to longitudinal vitamin A consumption and hypertensive status. This might be a micronutrient worth exploring in hypertensive animal models in order to further elucidate its protective role against oxidative stress.

The benefits of exercise and antioxidant intake in human chronic illnesses have been explored extensively, yet the effects of using both are still extremely controversial. A number of questions can be asked, such as: to what extent does antioxidant supplements and exercise interact and how does this interaction influence human endogenous antioxidant systems? Does timing of antioxidant intake and exercise matter? Are there significant differences in combining exercise and antioxidants in young adults and how about in individuals under more oxidative stress older adults, for example? How does one go about quantifying dietary intake of polyphenols and other antioxidants?

\section{References}

1. Basson M (2008) Cardiovascular disease. Nature 451: 903.

2. Poulter NR, Prabhakaran D, Caulfield M (2015) Hypertension. The Lancet 386: 801-812.

3. The Department of Health (2013) Cardiovascular disease. The Department of Health, Australian Government, Canberra, Australia.

4. World Health Organization (2013) A global brief on HYPERTENSION: Silent killer, global public health crisis. World Health Organization, Geneva, Switzerland.

5. Wray DW, Nishiyama SK, Donato AJ, Carlier P, Bailey DM, et al. (2011) The paradox of oxidative stress and exercise with advancing age. Exerc Sport Sci Rev 39: 68-76.

6. Draeger CL, Naves A, Marques N, Baptistella AB, Carnauba RA, et al. (2014) Controversies of antioxidant vitamins supplementation in exercise: ergogenic or ergolytic effects in humans? J Int Soc Sports Nutr 11: 4.

7. Alessio HM, Goldfarb AH, Cao G (1997) Exercise-induced oxidative stress before and after vitamin $\mathrm{C}$ supplementation. Int J Sport Nutr 7: 1-9.

8. Tauler P, Aguiló A, Gimeno I, Fuentespina E, Tur JA, et al. (2006) Response of blood cell antioxidant enzyme defences to antioxidant diet supplementation and to intense exercise. Eur J Nutr 45: 187-195.

9. Morillas-Ruiz JM, Villegas García JA, López FJ, Vidal-Guevara ML, Zafrilla $P$ (2006) Effects of polyphenolic antioxidants on exercise-induced oxidative stress. Clin Nutr 25: 444-453.

10. Manach C, Scalbert A, Morand C, Rémésy C, Jiménez L (2004) Polyphenols: food sources and bioavailability. Am J Clin Nutr 79: 727-747.

11. Ristow M, Zarse K, Oberbach A, Klöting N, Birringer M, et al. (2009) Antioxidants prevent health-promoting effects of physical exercise in humans. Proc Natl Acad Sci USA 106: 8665-8670.

12. Westerterp KR, Meijer EP (2002) Physical activity and oxidative stress in the elderly. Gerontechnology 2: 189-197.

13. Saito K, Yokoyama T, Yoshida H, Kim H, Shimada H, et al. (2012) A significant relationship between plasma vitamin $C$ concentration and physical performance among Japanese elderly women. J Gerontol A Biol Sci Med Sci 67: 295-301. 
14. Murase T, Haramizu S, Ota N, Hase T (2009) Suppression of the aging-associated decline in physical performance by a combination of resveratrol intake and habitual exercise in senescence-accelerated mice. Biogerontology 10 : 423-434.

15. Nalbant O, Toktaș N, Toraman NF, Ogüș C, Aydin H, et al. (2009) Vitamin E and aerobic exercise: effects on physical performance in older adults. Aging Clin Exp Res 21: 111-121.

16. Bobeuf F, Labonte M, Dionne IJ, Khalil A (2011) Combined effect of antioxidant supplementation and resistance training on oxidative stress markers, muscle and body composition in an elderly population. $J$ Nutr Health Aging 15: 883-889.

17. Llopis-González A, Rubio-López N, Pineda-Alonso M, Martín-Escudero JC Chaves FJ, et al. (2015) Hypertension and the Fat-soluble vitamins A, D and E. Int J Environ Res Public Health 12: 2793-2809.

18. Eskurza I, Monahan KD, Robinson JA, Seals DR (2004) Effect of acute and chronic ascorbic acid on flow-mediated dilatation with sedentary and physically active human ageing. J Physiol 556: 315-324.

19. Duffy SJ, Gokce N, Holbrook M, Hunter LM, Biegelsen ES, et al. (2001) Effect of ascorbic acid treatment on conduit vessel endothelial dysfunction in patients with hypertension. Am J Physiol Heart Circ Physiol 280: 528-534.

20. De la Fuente M, Hernanz A, Vallejo MC (2005) The immune system in the oxidative stress conditions of aging and hypertension: favorable effects of antioxidants and physical exercise. Antioxid Redox Signal 7: 1356-1366.

21. Godin G (2011) The Godin-Shephard Leisure-Time Physical Activity Questionnaire. Heal Fit J Canada 4: 18-22.

22. Barnard GA (1945) A New Test for $2 \times 2$ Tables. Nature 156: 177

23. Cleland CL, Hunter RF, Kee F, Cupples ME, Sallis JF, et al. (2014) Validity of the Global Physical Activity Questionnaire (GPAQ) in assessing levels and change in moderate-vigorous physical activity and sedentary behavior. BMC Public Health 14

24. Health Canada (2005) Dietary Reference Intakes Tables. Food and Nutrition, Health Canada, Ottawa, Canada.

25. Macdiarmid J, Blundell J (1998) Assessing dietary intake: Who, what and why of under-reporting. Nutr Res Rev 11: 231-253.

26. Garriguet D (2008) Under-reporting of energy intake in the Canadian Community Health Survey. Health Rep 19: 37-45

27. Ropponen A, Levalahti E, Simonen R, Videman T, Battie MC (2001) Repeatability of lifetime exercise reporting. Scand J Med Sci Sports 11: 185-192.
28. Williams L, Bradley L, Smith A, Foxwell B (2004) Signal transducer and activator of transcription 3 is the dominant mediator of the anti-inflammatory effects of IL-10 in human macrophages. J Immunol 172: 567-576.

29. Joachim G (1997) The influence of time on dietary data: differences in reported summer and winter food consumption. Nutr Health 12: 33-43.

30. Weder AB (2007) Genetics and hypertension. J Clin Hypertens (Greenwich) 9: $217-223$.

31. Agarwal A, Williams GH, Fisher ND (2005) Genetics of human hypertension. Trends Endocrinol Metab16: 127-133.

32. Coy V (2005) Genetics of essential hypertension. J Am Acad Nurse Pract 17: $219-224$

33. Habauzit V, Morand C (2012) Evidence for a protective effect of polyphenols-containing foods on cardiovascular health: an update for clinicians. Ther Adv Chronic Dis 3: 87-106.

34. Khurana S, Venkataraman K, Hollingsworth A, Piche M, Tai TC (2013) Polyphenols: benefits to the cardiovascular system in health and in aging. Nutrients 5: $3779-3827$.

35. Yang M, Wang Y, Davis CG, Lee SG, Fernandez ML, et al. (2014) Validation of an FFQ to assess short-term antioxidant intake against $30 \mathrm{~d}$ food records and plasma biomarkers. Public Health Nutr 17: 297-306.

36. Rautiainen S, Serafini M, Morgenstern R, Prior RL, Wolk A (2008) The validity and reproducibility of food-frequency questionnaire-based total antioxidant capacity estimates in Swedish women. Am J Clin Nutr 87: 1247-1253.

37. Pilgrim A, Robinson S, Sayer AA, Roberts H (2015) An overview of appetite decline in older people. Nurs Older People 27: 29-35.

38. Daley MJ, Spinks WL (2000) Exercise, mobility and aging. Sport Med 29: $1-12$.

39. Churchill JD, Galvez R, Colcombe S, Swain RA, Kramer AF, et al. (2002) Exercise, experience and the aging brain. Neurobiol Aging 23: 941-955.

40. Simpson RJ, Lowder TW, Spielmann G, Bigley AB, LaVoy EC, et al. (2012) Exercise and the aging immune system. Ageing Res Rev 11: 404-420.

41. Woods JA, Wilund KR, Martin SA, Kistler BM (2012) Exercise, inflammation and aging. Aging Dis 3: 130-140.

42. Crombie IK, Irvine L, Williams B, McGinnis AR, Slane PW, et al. (2004) Why older people do not participate in leisure time physical activity: A survey of activity levels, beliefs and deterrents. Age and Ageing 33: 287-292. 